\title{
ON TRUNCATIONS FOR WEAKLY ERGODIC INHOMOGENEOUS BIRTH AND DEATH PROCESSES
}

\author{
ALEXANDER ZEIFMAN ${ }^{*, * *, \dagger}$, YACOV SATIN ${ }^{* *}$, VICTOR KOROLEV $^{* * *, \dagger}$, SERGEY SHORGIN $^{\dagger}$ \\ *Institute of Socio-Economic Development of Territories \\ Russian Academy of Sciences, Gorkogo Str., 56A, Vologda, Russia \\ e-mail:a_zeifman@mail.ru \\ ** Department of Applied Mathematics \\ Vologda State University, Vologda, S. Orlova, 6, Russia \\ e-mail: yacovi@mail.ru \\ ***Faculty of Computational Mathematics and Cybernetics \\ Lomonosov Moscow State University, Leninskie Gory, Moscow, Russia \\ e-mail: victoryukorolev@yandex \\ ${ }^{\dagger}$ Institute of Problems of Informatics \\ Russian Academy of Sciences, Vavilova str., 44-2, Moscow, Russia
}

\begin{abstract}
We investigate a class of exponentially weakly ergodic inhomogeneous birth and death processes. We consider special transformations of the reduced intensity matrix of the process and obtain uniform (in time) error bounds of truncations. Our approach also guarantees that we can find limiting characteristics approximately with an arbitrarily fixed error. As an example, we obtain the respective bounds of the truncation error for an $M_{t} / M_{t} / S$ queue for any number of servers $S$. Arbitrary intensity functions instead of periodic ones can be considered in the same manner.
\end{abstract}

Keywords: birth and death process, weak ergodicity, truncation, forward Kolmogorov system, nonstationary Markovian queueing models.

\section{Introduction}

It is well known that explicit expressions for probability characteristics of stochastic models can be found only in a few special cases. In view of this, the study of the rate of convergence as time $t \rightarrow \infty$ to the steady state of a process is one of two main problems for obtaining the limiting behaviour of the process. If the model is Markovian and stationary in time, then the stationary limiting characteristics usually give usually, sufficient or almost sufficient information about the model. On the other hand, if we deal with an inhomogeneous Markovian model, then we must approximately calculate, in addition to that, the limiting probability characteristics of the process. The problem of existence and construction of limiting characteristics for inhomogeneous (in time) birth and death processes is important for queueing applications (see, e.g., Di Crescenzo and Nobile, 1995; Di Crescenzo et al., 2003; 2012; Granovsky and Zeifman, 2004;
Mandelbaum and Massey, 1995; Massey and Whitt, 1994; Massey and Pender, 2013; Olwal et al., 2012; Tan et al., 2013; Zeifman et al., 2006). This is the second main problem. A general approach and related bounds for the study on the convergence rate was considered by Zeifman (1995a), who also first mentioned computation of the limiting characteristics for the process via truncations (Zeifman, 1988), and later considered it in detail (Zeifman et al., 2006). The first results for more general Markovian queueing models have been obtained recently by Zeifman et al. (2014).

About two decades ago Vladimir V. Kalashnikov suggested that in some cases one can obtain uniform (in time) error bounds of truncation. Here we prove this conjecture.

The paper is organized as follows. Basic notions are recalled below in Section 1. Auxiliary statements are considered in Section 2. The main result is proved in 
Section 3. In Section 4 we consider application of our bounds for the $M_{t} / M_{t} / S$ queueing model. Finally, in Section 5 we consider a specific queueing example.

Let $X=X(t), t \geq 0$ be a Birth and Death Process (BDP) with birth and death rates $\lambda_{n}(t), \mu_{n}(t)$, respectively. Let $p_{i j}(s, t)=\operatorname{Pr}\{X(t)=j \mid X(s)=i\}$ for $i, j \geq 0,0 \leq s \leq t$, be the transition probability functions of the process $X=X(t)$, and let $p_{i}(t)=$ $\operatorname{Pr}\{X(t)=i\}$ be the state probabilities.

We assume throughout the paper that

$$
\begin{aligned}
\operatorname{Pr} & (X(t+h)=j / X(t)=i) \\
& = \begin{cases}q_{i j}(t) h+\alpha_{i j}(t, h) & \text { if } j \neq i, \\
1-\sum_{k \neq i} q_{i k}(t) h+\alpha_{i}(t, h) & \text { if } j=i,\end{cases}
\end{aligned}
$$

where all $\alpha_{i}(t, h)$ are $o(h)$ uniformly in $i$, i.e., $\sup _{i}\left|\alpha_{i}(t, h)\right|=o(h)$. Here all $q_{i, i+1}(t)=\lambda_{i}(t)$, $q_{i, i-1}(t)=\mu_{i}(t)$ for any $i>0$, and all other $q_{i j}(t) \equiv 0$.

The probabilistic dynamics of the process are represented by the forward Kolmogorov system of differential equations:

$$
\left\{\begin{array}{l}
\frac{\mathrm{d} p_{0}}{\mathrm{~d} t}=-\lambda_{0}(t) p_{0}+\mu_{1}(t) p_{1}, \\
\frac{\mathrm{d} p_{k}}{\mathrm{~d} t}=\lambda_{k-1}(t) p_{k-1}-\left(\lambda_{k}(t)+\mu_{k}(t)\right) p_{k} \\
+\mu_{k+1}(t) p_{k+1}, \quad k \geq 1 .
\end{array}\right.
$$

By $\mathbf{p}(t)=\left(p_{0}(t), p_{1}(t), \ldots\right)^{T}, t \geq 0$, we denote the column vector of state probabilities and by $A(t)=$ $\left(a_{i j}(t)\right), t \geq 0$, the matrix related to (2). One can see that $A(t)=Q^{T}(t)$, where $Q(t)$ is the intensity (or infinitesimal) matrix for $X(t)$.

We assume that all birth and death intensity functions $\lambda_{i}(t)$ and $\mu_{i}(t)$ are linear combinations of a finite number of functions which are locally integrable on $[0, \infty)$. Moreover, we assume that

$$
\lambda_{n}(t) \leq L<\infty, \quad \mu_{n}(t) \leq M<\infty,
$$

for almost all $t \geq 0$. Throughout the paper by $\|\cdot\|$ we denote the $l_{1}$-norm, i.e., $\|\mathbf{x}\|=\sum\left|x_{i}\right|$, and $\|B\|=$ $\sup _{j} \sum_{i}\left|b_{i j}\right|$ for $B=\left(b_{i j}\right)_{i, j=0}^{\infty}$.

Let $\Omega$ be a set all stochastic vectors, i.e., $l_{1}$ vectors with nonnegative coordinates and the unit norm. Then we have

$$
\|A(t)\| \leq 2 \sup \left(\lambda_{k}(t)+\mu_{k}(t)\right) \leq 2(L+M)
$$

for almost all $t \geq 0$. Hence the operator function $A(t)$ from $l_{1}$ into itself is bounded for almost all $t \geq 0$ and locally integrable on $[0 ; \infty)$.

Therefore we can consider the system (2) as the differential equation

$$
\frac{\mathrm{d} \mathbf{p}}{\mathrm{d} t}=A(t) \mathbf{p}, \quad \mathbf{p}=\mathbf{p}(t), \quad t \geq 0,
$$

in the space $l_{1}$ with a bounded operator function $A(t)$.

It is well known (Daleckij and Krein, 1974) that the Cauchy problem for the differential equation (1) has unique solutions for arbitrary initial conditions, and $\mathbf{p}(s) \in \Omega$ implies $\mathbf{p}(t) \in \Omega$ for $t \geq s \geq 0$.

Therefore, we can apply the general approach to employ the logarithmic norm of a matrix for the study of the stability of a Kolmogorov system of differential equations associated with nonhomogeneous Markov chains. The method is based on the following two components: the logarithmic norm of a linear operator and a special similarity transformation of the matrix of intensities of the Markov chain considered, see the corresponding definitions, bounds, references and other details in the works of Van Doorn et al. (2010), Granovsky and Zeifman (2004), Zeifman (1985; 1995b; 1995a) or Zeifman et al. (2006).

Definition 1. A Markov chain $X(t)$ is called weakly ergodic if $\left\|\mathbf{p}^{*}(t)-\mathbf{p}^{* *}(t)\right\| \rightarrow 0$ as $t \rightarrow \infty$ for any initial conditions $\mathbf{p}^{*}(0), \mathbf{p}^{* *}(0)$. Here $\mathbf{p}^{*}(t)$ and $\mathbf{p}^{* *}(t)$ are the corresponding solutions of (4).

Set $E_{k}(t)=E\{X(t) \mid X(0)=k\}$ (then the corresponding initial condition of the system (4) is the $k$-th unit vector $\mathbf{e}_{\mathbf{k}}$ ).

Definition 2. Let $X(t)$ be a Markov chain. Then $\varphi(t)$ is called the limiting mean of $X(t)$ if

$$
\lim _{t \rightarrow \infty}\left(\varphi(t)-E_{k}(t)\right)=0
$$

for any $k$.

\section{Auxiliary notions and results}

Consider an increasing sequence of positive numbers $\left\{d_{i}\right\}, i=1,2, \ldots, d_{1}=1$, and the corresponding triangular matrix

$$
D=\left(\begin{array}{cccc}
d_{1} & d_{1} & d_{1} & \cdots \\
0 & d_{2} & d_{2} & \cdots \\
0 & 0 & d_{3} & \cdots \\
& \ddots & \ddots & \ddots
\end{array}\right)
$$

Let $l_{1 D}$ be the space of sequences

$$
l_{1 D}=\left\{\mathbf{z}=\left(p_{1}, p_{2}, \ldots\right)^{T}:\|\mathbf{z}\|_{1 D} \equiv\|D \mathbf{z}\|<\infty\right\} .
$$

We also introduce the auxiliary space of sequences $l_{1 E}$ as

$$
l_{1 E}=\left\{\mathbf{z}=\left(p_{1}, p_{2}, \cdots\right)^{T}:\|\mathbf{z}\|_{1 E} \equiv \sum k\left|p_{k}\right|<\infty\right\} .
$$

Set

$$
d=\inf _{i \geq 1} d_{i}=1, \quad W=\inf _{i \geq 1} \frac{d_{i}}{i}, \quad g_{i}=\sum_{n=1}^{i} d_{n} .
$$


Consider the following expressions:

$$
\begin{aligned}
\alpha_{k}(t)= & \lambda_{k}(t)+\mu_{k+1}(t)-\frac{d_{k+1}}{d_{k}} \lambda_{k+1}(t) \\
& -\frac{d_{k-1}}{d_{k}} \mu_{k}(t), \quad k \geq 0,
\end{aligned}
$$

and

$$
\alpha(t)=\inf _{k \geq 0} \alpha_{k}(t)
$$

At first, we recall the definition of the logarithmic norm and the related bound (for details, see Van Doorn et al., 2010; Granovsky and Zeifman, 2004; Zeifman et al., 2006)

Let $B(t), t \geq 0$ be a one-parameter family of bounded linear operators on a Banach space $\mathcal{B}$ and let $I$ denote the identity operator. For a given $t \geq 0$, the number

$$
\gamma(B(t))_{\mathcal{B}}=\lim _{h \rightarrow+0} \frac{\|I+h B(t)\|-1}{h}
$$

is called the logarithmic norm of the operator $B(t)$.

If $\mathcal{B}$ is an $(N+1)$-dimensional vector space with the $l_{1}$-norm, so that the operator $B(t)$ is given by the matrix $B(t)=\left(b_{i j}(t)\right)_{i, j=0}^{N}, t \geq 0$, then the logarithmic norm of $B(t)$ can be found explicitly:

$$
\gamma(B(t))=\sup _{j}\left(b_{j j}(t)+\sum_{i \neq j}\left|b_{i j}(t)\right|\right), \quad t \geq 0 .
$$

On the other hand, the logarithmic norm of the operator $B(t)$ is related to the Cauchy operator $V(t, s)$ of the system

$$
\frac{\mathrm{d} \mathbf{x}}{\mathrm{d} t}=B(t) \mathbf{x}, \quad t \geq 0
$$

in the following way:

$$
\gamma(B(t))_{\mathcal{B}}=\lim _{h \rightarrow+0} \frac{\|V(t+h, t)\|-1}{h}, \quad t \geq 0 .
$$

From the latter, one can deduce the following bounds of the Cauchy operator $V(t, s)$ :

$$
\|V(t, s)\|_{\mathcal{B}} \leq e^{\int^{t} \gamma(B(\tau)) \mathrm{d} \tau}, \quad 0 \leq s \leq t .
$$

Recall now the following general statement.

Theorem 1. Let a BDP with the rates $\lambda_{k}(t)$ and $\mu_{k}(t)$ be given. Assume that there exists a sequence $\left\{d_{i}\right\}$ such that

$$
\int_{0}^{\infty} \alpha(t) \mathrm{d} t=+\infty
$$

Then $X(t)$ is weakly ergodic, and the following bounds hold:

$$
\begin{aligned}
& \left\|\mathbf{p}^{*}(t)-\mathbf{p}^{* *}(t)\right\|_{1 D} \\
& \quad \leq e^{-\int_{s}^{t} \alpha(\tau) \mathrm{d} \tau}\left\|\mathbf{p}^{*}(s)-\mathbf{p}^{* *}(s)\right\|_{1 D},
\end{aligned}
$$

$$
\begin{aligned}
\| \mathbf{p}^{*}(t)- & \mathbf{p}^{* *}(t) \| \\
& \leq 4 e^{-\int_{s}^{t} \alpha(\tau) \mathrm{d} \tau} \sum_{i \geq 1} g_{i}\left|p_{i}^{*}(s)-p_{i}^{* *}(s)\right|,
\end{aligned}
$$

for any $t \geq s \geq 0$ and any initial conditions $\mathbf{p}^{*}(s)$ and $\mathbf{p}^{* *}(s)$.

Proof. The property $\mathbf{p}(t) \in \Omega$ for any $t \geq 0$ allows putting $p_{0}(t)=1-\sum_{i \geq 1} p_{i}(t)$. Then from (4) we obtain the following system:

$$
\frac{\mathrm{d} \mathbf{z}(t)}{\mathrm{d} t}=B(t) \mathbf{z}(t)+\mathbf{f}(t)
$$

where

$$
\begin{gathered}
\mathbf{z}(\mathbf{t})=\left(p_{1}(t), p_{2}(t), \ldots\right)^{T}, \\
\mathbf{f}(\mathbf{t})=\left(\lambda_{0}(t), 0,0, \ldots\right)^{T}, \\
B(t)=\left(b_{i j}(t)\right)_{i, j=1}^{\infty}
\end{gathered}
$$

and

$$
b_{i j}= \begin{cases}-\left(\lambda_{0}+\lambda_{1}+\mu_{1}\right) & \text { if } i=j=1, \\ \mu_{2}-\lambda_{0} & \text { if } i=1, j=2, \\ -\lambda_{0} & \text { if } i=1, j>2, \\ -\left(\lambda_{j}+\mu_{j}\right) & \text { if } i=j>1 \\ \mu_{j} & \text { if } i=j-1>1, \\ \lambda_{j} & \text { if } i=j+1>1, \\ 0 & \text { otherwise. }\end{cases}
$$

This is a linear non-homogeneous differential system the solution of which can be written as

$$
\mathbf{z}(t)=V(t, 0) \mathbf{z}(0)+\int_{0}^{t} V(t, \tau) \mathbf{f}(\tau) \mathrm{d} \tau,
$$

where $V(t, z)$ is the Cauchy operator of (11) (see, e.g., Zeifman, 1995a).

Consider Eqn. (11) in the space $l_{1 D}$. We have $\|\mathbf{f}(t)\|_{1 D}=d_{1} \lambda_{0}(t) \leq L$ for almost all $t \geq 0$. On the 
other hand,

$$
D B D^{-1}=\left(\begin{array}{ccc}
-\left(\lambda_{0}+\mu_{1}\right) & \frac{d_{1}}{d_{2}} \mu_{2} \\
\frac{d_{2}}{d_{1}} \lambda_{1} & -\left(\lambda_{1}+\mu_{2}\right) \\
0 & \frac{d_{3}}{d_{2}} \lambda_{2} \\
& \ddots \\
& & \\
0 & \ddots & \\
\frac{d_{2}}{d_{3}} \mu_{3} & 0 & \ddots \\
-\left(\lambda_{2}+\mu_{3}\right) & \frac{d_{3}}{d_{4}} \mu_{4} & 0 \\
\ddots & \ddots & \\
\ddots & \ddots & \ddots
\end{array}\right) .
$$

Hence

$$
\begin{aligned}
\| & B(t) \|_{1 D} \\
= & \sup _{k \geq 0}\left(\lambda_{k}(t)+\mu_{k+1}(t)+\frac{d_{k+1}}{d_{k}} \lambda_{k+1}(t)\right. \\
& \left.+\frac{d_{k-1}}{d_{k}} \mu_{k}(t)\right) \\
\leq & \sup _{k \geq 0}\left(\left(-\lambda_{k}(t)-\mu_{k+1}(t)+\frac{d_{k+1}}{d_{k}} \lambda_{k+1}(t)\right.\right. \\
& \left.\left.+\frac{d_{k-1}}{d_{k}} \mu_{k}(t)\right)+2 \lambda_{k}(t)+2 \mu_{k+1}(t)\right) \\
\leq & 2(L+M)-\alpha(t)
\end{aligned}
$$

for almost all $t \geq 0$.

Then $\mathbf{f}(t)$ and $B(t)$ are bounded and locally integrable on $[0, \infty)$ as a vector function and an operator function in $l_{1 D}$, respectively.

Now we have the following bound for the logarithmic norm $\gamma(B(t))$ in $l_{1 D}$ :

$$
\begin{aligned}
\gamma(B)_{1 D}= & \gamma\left(D B(t) D^{-1}\right)_{1} \\
= & \sup _{i \geq 0}\left(\frac{d_{i+1}}{d_{i}} \lambda_{i+1}(t)+\frac{d_{i-1}}{d_{i}} \mu_{i}(t)\right. \\
& \left.-\left(\lambda_{i}(t)+\mu_{i+1}(t)\right)\right) \\
= & -\inf _{k \geq 0}\left(\alpha_{k}(t)\right)=-\alpha(t),
\end{aligned}
$$

in accordance with (7). Hence

$$
\|V(t, s)\|_{1 D} \leq e^{-\int_{s}^{t} \alpha(\tau) \mathrm{d} \tau} .
$$

Therefore, the bound (9) holds.
On the other hand, we have

$$
\begin{aligned}
& \|\mathbf{z}\| \\
& \leq \sum_{i \geq 1} d_{i}\left|p_{i}\right| \\
& =d_{1}\left(\left|\sum_{i \geq 1} p_{i}+\sum_{i \geq 2}-p_{i}\right|\right) \\
& \quad+d_{2}\left(\left|\sum_{i \geq 2} p_{i}+\sum_{i \geq 3}-p_{i}\right|\right)+\ldots \\
& \leq d_{1}\left|\sum_{i \geq 1} p_{i}\right|+2 d_{2}\left|\sum_{i \geq 2} p_{i}\right|+\cdots \leq 2\|\mathbf{z}\|_{1 D},
\end{aligned}
$$

and $\left\|\mathbf{p}^{*}-\mathbf{p}^{* *}\right\| \leq 2\|\mathbf{z}\|$ for any $\mathbf{p}^{*}, \mathbf{p}^{* *}$ and the corresponding $\mathbf{z}$. Hence the bound (10) holds.

Corollary 1. Let, in addition, the numbers $d_{i}$ grow sufficiently fast so that $W>0$. Then $X(t)$ has the limiting mean, say $\phi(t)$, and the following bound holds:

$$
\left|\phi(t)-E_{k}(t)\right| \leq \frac{4}{W} e^{-\int_{0}^{t} \alpha(\tau) \mathrm{d} \tau}\left\|\mathbf{p}(0)-\mathbf{e}_{\mathbf{k}}\right\|_{1 D} .
$$

Proof. The bound (19) follows from (9) and from the inequality

$$
\begin{aligned}
\|\mathbf{z}\|_{1 D} & =d_{1}\left|\sum_{i=1}^{\infty} p_{i}\right|+d_{2}\left|\sum_{i=2}^{\infty} p_{i}\right|+\ldots \\
& \geq W \sum_{k \geq 1} k\left|\sum_{i \geq k} p_{i}\right| \geq \frac{W}{2}\|\mathbf{z}\|_{1 E} .
\end{aligned}
$$

\section{Truncations}

Now we consider the family of "truncated" processes $X_{N}(t)$ on the state space $E_{N}=\{0,1, \ldots, N\}$, where the birth rates are $\lambda_{n}(t), n \in E_{N-1}$ and the death rates are $\mu_{n}(t), n \in E_{N}$ (and with the intensity matrix $A_{N}$ ). The truncated process has the vector of probabilities governed by the forward Kolmogorov differential system

$$
\frac{\mathrm{d} \mathbf{p}_{N}}{\mathrm{~d} t}=A_{N}(t) \mathbf{p}_{N} .
$$

Below we will identify the finite vector with entries $\left(a_{1}, \ldots, a_{N}\right)$ and the infinite vector with the same first $N$ coordinates and the others equal to zero. The same identification will be assumed also for the rate matrix $A_{N}$, triangular matrix $D_{N}$, and so on.

Theorem 2. Let a BDP with the rates $\lambda_{k}(t)$ and $\mu_{k}(t)$ be given. Assume that there exists a sequence $\left\{d_{i}\right\}$ such that

$$
\int_{0}^{\infty} \alpha(t) \mathrm{d} t=+\infty
$$


Then $X_{N}(t)$ is weakly ergodic for any $N$, and the following bounds hold:

$$
\begin{aligned}
& \left\|\mathbf{p}_{N}^{*}(t)-\mathbf{p}^{* *}{ }_{N}(t)\right\|_{1 D} \\
& \leq e^{-\int_{s}^{t} \alpha(\tau) \mathrm{d} \tau}\left\|\mathbf{p}_{N}^{*}(s)-\mathbf{p}^{* *}{ }_{N}(s)\right\|_{1 D}, \\
& \left\|\mathbf{p}_{N}^{*}(t)-\mathbf{p}^{* *}{ }_{N}(t)\right\| \\
& \leq 4 e^{-\int_{s}^{t} \alpha(\tau) d \tau} \sum_{i \geq 1} g_{i}\left|p_{i, N}^{*}(s)-p_{i, N}^{* *}(s)\right| \\
& \leq 8 g_{N} e^{-\int_{s}^{t} \alpha(\tau) d \tau},
\end{aligned}
$$

for any $t \geq s \geq 0$ and any initial conditions $\mathbf{p}^{*}{ }_{N}(s)$ and $\mathbf{p}^{* *}{ }_{N}(s)$.

Proof. The property $\mathbf{p}_{N}(t) \in \Omega$ for any $t \geq 0$ allows setting $p_{0, N}(t)=1-\sum_{i>1} p_{i, N}(t)$. Then from (21) we obtain the following system:

$$
\frac{\mathrm{d} \mathbf{z}_{N}(t)}{\mathrm{d} t}=B_{N}(t) \mathbf{z}_{N}(t)+\mathbf{f}_{N}(t),
$$

where $\mathbf{z}_{\mathbf{N}}(\mathbf{t})=\left(p_{1, N}(t), \ldots, p_{N, N}(t)\right)^{T}, \mathbf{f}_{\mathbf{N}}(\mathbf{t})=$ $\left(\lambda_{0}(t), 0, \ldots, 0\right)^{T}, B_{N}(t)=\left(b_{i j}(t)\right)_{i, j=1}^{N}$ and

$b_{i j}= \begin{cases}-\left(\lambda_{0}+\lambda_{1}+\mu_{1}\right) & \text { if } i=j=1 \\ \mu_{2}-\lambda_{0} & \text { if } i=1, j=2 \\ -\lambda_{0} & \text { if } i=1, j>2 \\ -\left(\lambda_{j}+\mu_{j}\right) & \text { if } i=j>1 \\ \mu_{j} & \text { if } 1<i=j-1 \leq N \\ \lambda_{j} & \text { if } 1<i=j+1 \leq N-1 \\ 0 & \text { otherwise. }\end{cases}$

The solution of Eqn. 25) can be written as

$$
\mathbf{z}_{N}(t)=V_{N}(t, 0) \mathbf{z}_{N}(0)+\int_{0}^{t} V_{N}(t, \tau) \mathbf{f}_{N}(\tau) \mathrm{d} \tau,
$$

where $V_{N}(t, z)$ is the Cauchy operator of (25).

Now the following bound for the logarithmic norm $\gamma\left(B_{N}(t)\right)$ in the $l_{1 D, N}$ norm holds:

$$
\begin{aligned}
\gamma\left(B_{N}\right)_{1 D, N} \\
=\gamma\left(D_{N} B_{N}(t) D_{N}^{-1}\right)_{1} \\
\leq \max _{0 \leq i \leq N-1}\left(\frac{d_{i+1}}{d_{i}} \lambda_{i+1}(t)+\frac{d_{i-1}}{d_{i}} \mu_{i}(t)\right. \\
\left.\quad-\left(\lambda_{i}(t)+\mu_{i+1}(t)\right)\right) \\
\leq \sup _{k \geq 0}\left(-\alpha_{k}(t)\right)=-\alpha(t)
\end{aligned}
$$

in accordance with 25). Hence

$$
\left\|V_{N}(t, s)\right\|_{1 D, N} \leq e^{-\int_{s}^{t} \alpha(\tau) \mathrm{d} \tau} .
$$

Our claim follows from this bound.
Denote by $E_{k, N}(t)=E\left\{X_{N}(t) \mid X_{N}(0)=k\right\}$ the mathematical expectation of the truncated process under the initial condition $\mathbf{e}_{\mathbf{k}}$.

Corollary 2. Under the assumptions of Theorem 2 the truncated process $X_{N}(t)$ has the limiting mean for any $N$, say $\phi_{N}(t)$, and the following bounds hold:

$$
\begin{aligned}
\mid \phi_{N}(t)- & E_{k, N}(t) \mid \\
& \leq \frac{4}{W} e^{-\int_{0}^{t} \alpha(\tau) \mathrm{d} \tau}\left\|\mathbf{p}_{N}(0)-\mathbf{e}_{\mathbf{k}}\right\|_{1 D, N},
\end{aligned}
$$

and

$$
\left|\phi_{N}(t)-E_{k, N}(t)\right| \leq 8 N g_{N},
$$

for any $k$ and any $t \geq 0$.

Now if we suppose in addition that there exist positive $R$ and $a$ such that

$$
e^{-\int_{s}^{t} \alpha(u) \mathrm{d} u} \leq R e^{-a(t-s)}
$$

for any $0 \leq s \leq t$, then the process $X(t)$ is exponentially weakly ergodic.

Moreover, consider a "new" sequence $\left\{d_{i}^{*}\right\}$ such that $d_{i}^{*}=d_{i}^{2}$, and the correspondent inequality

$$
e^{-\int_{s}^{t} \alpha^{*}(u) \mathrm{d} u} \leq R^{*} e^{-a^{*}(t-s)}
$$

for any $0 \leq s \leq t$.

Theorem 3. Let the assumptions of Theorem 2 hold. In addition, let the inequalities (32) and (33) hold for some positive $R, R^{*}, a, a^{*}$. Then we have the following bounds of truncation:

$$
\left\|\mathbf{p}(t)-\mathbf{p}_{N}(t)\right\| \leq \frac{8 L R R^{*}(L+M)}{a a^{*} d_{N}}
$$

for $\mathbf{p}(0)=\mathbf{p}_{N}(0)=\mathbf{e}_{\mathbf{0}}$,

$$
\left|E_{0}(t)-E_{0, N}(t)\right| \leq \frac{4 L R R^{*}(L+M)}{a a^{*} d_{N} W} .
$$

Proof. Consider the correspondent non-homogeneous equation for $X(t)$ (11) in the form

$$
\frac{\mathrm{d} \mathbf{z}(t)}{\mathrm{d} t}=B_{N}(t) \mathbf{z}(t)+\left(B(t)-B_{N}(t)\right) \mathbf{z}(t)+\mathbf{f}(t) .
$$

Then we have

$$
\begin{aligned}
\mathbf{z}(t)= & V_{N}(t) \mathbf{z}(0) \\
& +\int_{0}^{t} V_{N}(t, \tau)\left(B(\tau)-B_{N}(\tau)\right) \mathbf{z}(\tau) \mathrm{d} \tau \\
& +\int_{0}^{t} V_{N}(t, \tau) \mathbf{f}(\tau) \mathrm{d} \tau
\end{aligned}
$$


We note that the following equality holds:

$$
V_{N}(t, s) \mathbf{f}(s)=V_{N}(t, s) \mathbf{f}_{\mathbf{N}}(s)
$$

for any $0 \leq s \leq t$. Hence we have

$$
\begin{aligned}
\mathbf{z}(t)- & \mathbf{z}_{N}(t) \\
& =\int_{0}^{t} V_{N}(t, \tau)\left(B(\tau)-B_{N}(\tau)\right) \mathbf{z}(\tau) \mathrm{d} \tau
\end{aligned}
$$

if $\mathbf{p}(0)=\mathbf{p}_{N}(0)$.

Write $B(t)-B_{N}(t)=\left(b_{i j}^{*}(t)\right)_{i, j=1}^{\infty}$. Then

$$
b_{i j}^{*}= \begin{cases}0 & \text { if } i=1, j \leq N \\ -\lambda_{0} & \text { if } i=1, j>N \\ 0 & \text { if } 2 \leq i \leq N-1, j \leq 1 \\ -\lambda_{N} & \text { if } i=j=N \\ -\left(\lambda_{j}+\mu_{j}\right) & \text { if } i=j>N \\ \mu_{j} & \text { if } i \geq N, j=i+1 \\ \lambda_{j} & \text { if } N>i, j=i-1 \\ 0 & \text { otherwise. }\end{cases}
$$

Hence

$$
\left(B(\tau)-B_{N}(\tau)\right) \mathbf{z}(\tau)=\left(r_{1}(\tau), r_{2}(\tau), \ldots\right)^{T}
$$

where

$$
r_{i}= \begin{cases}-\lambda_{0} \sum_{i>N} p_{i} & \text { if } i=1, \\ 0 & \text { if } 2 \leq i \leq N-1, \\ -\lambda_{N} p_{N}+\mu_{N+1} p_{N+1} & \text { if } i=N, \\ \lambda_{i-1} p_{i-1}-\left(\lambda_{i}+\mu_{i}\right) p_{i} & \\ +\mu_{i+1} p_{i+1} & \text { if } i>N .\end{cases}
$$

Now we have

$$
D\left(B(\tau)-B_{N}(\tau)\right) \mathbf{z}(\tau)=\left(r_{1}^{*}(\tau), r_{2}^{*}(\tau), \ldots\right)^{T},
$$

where

$$
r_{i}^{*}= \begin{cases}-d_{1} \lambda_{0} \sum_{i>N} p_{i} & \text { if } i=1, \\ 0 & \text { if } 2 \leq i \leq N \\ d_{i}\left(\lambda_{i} p_{j}-\mu_{i+1} p_{i+1}\right) & \text { if } i>N\end{cases}
$$

and

$$
\begin{aligned}
& \left\|\left(B(\tau)-B_{N}(\tau)\right) \mathbf{z}(\tau)\right\|_{1 D} \\
& \quad=\sum_{i}\left|r_{i}^{*}(\tau)\right| \\
& \quad \leq 2(L+M) \sum_{i=N}^{\infty} d_{i} p_{i}(\tau) .
\end{aligned}
$$

Therefore, in the $l_{1 D}$-norm the following bound holds:

$$
\begin{aligned}
& \left\|\mathbf{z}(t)-\mathbf{z}_{N}(t)\right\| \\
& \leq \int_{0}^{t}\left\|V_{N}(t, \tau)\right\|\left\|\left(B(\tau)-B_{N}(\tau)\right) \mathbf{z}(\tau)\right\| \mathrm{d} \tau \\
& \leq 2(L+M) \int_{0}^{t} R e^{-a(t-\tau)} \sum_{i=N}^{\infty} \mathrm{d}_{i} p_{i}(\tau) \mathrm{d} \tau \\
& \leq \frac{2 R(L+M)}{a} \sup _{0 \leq \tau \leq t} \sum_{i=N}^{\infty} \mathrm{d}_{i} p_{i}(\tau) .
\end{aligned}
$$

For estimating the right-hand side of 45 we consider now the upper and lower bounds for $\|\mathbf{z}(t)\|_{1 D^{*}}$. Firstly, one has

$$
\begin{aligned}
& \|\mathbf{z}(t)\|_{1 D^{*}} \\
& \leq\|V(t)\|_{1 D^{*}}\|\mathbf{z}(0)\|_{1 D^{*}} \\
& \quad+\int_{0}^{t}\|V(t, \tau)\|_{1 D^{*}}\|\mathbf{f}(\tau)\|_{1 D^{*}} \mathrm{~d} \tau \\
& \leq R^{*} e^{-a^{*} t}\|\mathbf{z}(0)\|_{1 D^{*}}+\frac{L R^{*}}{a^{*}}
\end{aligned}
$$

since $\lambda_{0}(t) \leq L$ for almost all $t \geq 0$.

Set $X(0)=0$. Then $\mathbf{p}(0)=(1,0,0, \ldots)^{T}, \mathbf{z}(0)=$ $\mathbf{0}$, and hence

$$
\|\mathbf{z}(t)\|_{1 D^{*}} \leq \frac{L R^{*}}{a^{*}}
$$

for any $t \geq 0$.

On the other hand, all $p_{i}(t) \geq 0$. Therefore

$$
\begin{aligned}
& \|\mathbf{z}(t)\|_{1 D^{*}} \\
& \quad=\sum_{i \geq 1} p_{i}(t) \sum_{k=1}^{i} d_{k}^{2} \geq \sum_{i \geq N} d_{i}^{2} p_{i}(t) \\
& \quad \geq d_{N} \sum_{i \geq N} d_{i} p_{i}(t),
\end{aligned}
$$

and

$$
\sum_{i=N}^{\infty} d_{i} p_{i}(t) \leq \frac{\|\mathbf{z}(t)\|_{1 D^{*}}}{d_{N}} \leq \frac{L R^{*}}{a^{*} d_{N}},
$$

for any $t \geq 0$.

Finally, we have

$$
\left\|\mathbf{z}(t)-\mathbf{z}_{N}(t)\right\|_{1 D} \leq \frac{2 L R R^{*}(L+M)}{a a^{*} d_{N}},
$$

for $\mathbf{p}(0)=\mathbf{p}_{N}(0)=\mathbf{e}_{\mathbf{0}}$, and the first bound of our theorem follows now from the inequality $d\|\mathbf{z}\| \leq 2\|\mathbf{z}\|_{1 D}$. The inequality

$$
\begin{aligned}
\|\mathbf{z}\|_{1 D} & =d_{1}\left|\sum_{i=1}^{\infty} p_{i}\right|+d_{2}\left|\sum_{i=2}^{\infty} p_{i}\right|+\ldots \\
& \geq W \sum_{k \geq 1} k\left|\sum_{i \geq k} p_{i}\right| \geq \frac{W}{2}\|\mathbf{z}\|_{1 E}
\end{aligned}
$$

implies the second bound. 
Denote by $\mathbf{p}(t, k)$ the probability distribution of $X(t)$ with the initial condition $\mathbf{p}(0, k)=\mathbf{e}_{\mathbf{k}}$, i.e., with the initial condition $X(0)=k$.

Theorem 4. Under the assumptions of Theorem 3 the following bounds hold:

$$
\begin{array}{r}
\left\|\mathbf{p}(t, k)-\mathbf{p}_{N}(t, 0)\right\| \\
\leq 8 R g_{k} e^{-a t}+\frac{8 L R R^{*}(L+M)}{a a^{*} d_{N}}, \\
\begin{array}{r}
\left\|\mathbf{p}(t, k)-\mathbf{p}_{N}(t, 0)\right\| \\
\leq 8 R^{*} g_{k}^{*} e^{-a^{*} t}+\frac{8 L R R^{*}(L+M)}{a a^{*} d_{N}}
\end{array}
\end{array}
$$

and

$$
\begin{aligned}
& \left|E_{k}(t)-E_{0, N}(t)\right| \\
& \leq \frac{4 R g_{k}}{W} e^{-a t}+\frac{4 L R R^{*}(L+M)}{a a^{*} d_{N} W}, \\
& \left|E_{k}(t)-E_{0, N}(t)\right| \\
& \leq \frac{4 R^{*} g_{k}^{*}}{W^{*}} e^{-a^{*} t}+\frac{4 L R R^{*}(L+M)}{a a^{*} d_{N} W} .
\end{aligned}
$$

Proof. The claim follows from the inequalities (10), 19, (34) and (35).

We formulate separately the correspondent statement for the important case of periodic (1-periodic, for definiteness) intensities.

Note firstly that in this situation the condition (22) implies the inequality (32) for

$a=\int_{0}^{1} \alpha(t) \mathrm{d} t, \quad R=e^{K}, \quad K=\sup _{|t-s| \leq 1} \int_{s}^{t} \alpha(u) \mathrm{d} u$.

Similarly, the assumption

$$
\int_{0}^{\infty} \alpha^{*}(t) \mathrm{d} t=\infty
$$

implies the inequality (33) for

$$
\begin{aligned}
a^{*} & =\int_{0}^{1} \alpha^{*}(t) \mathrm{d} t, \\
R^{*} & =e^{K^{*}}, \\
K^{*} & =\sup _{|t-s| \leq 1} \int_{s}^{t} \alpha^{*}(u) \mathrm{d} u .
\end{aligned}
$$

Theorem 5. Under the assumptions of Theorem 3 let the birth and death intensities be 1-periodic. Then there exist a limiting 1-periodic probability distribution $\pi(t)$, say, and the respective limiting 1-periodic mean $\phi(t)$, and the following bounds hold:

$$
\begin{array}{r}
\left\|\pi(t)-\mathbf{p}_{N}(t, 0)\right\| \\
\leq \frac{4 L R^{2}}{a} e^{-a t}+\frac{8 L R R^{*}(L+M)}{a a^{*} d_{N}}, \\
\left\|\pi(t)-\mathbf{p}_{N}(t, 0)\right\| \\
\leq \frac{4 L R^{* 2}}{a^{*}} e^{-a^{*} t}+\frac{8 L R R^{*}(L+M)}{a a^{*} d_{N}},
\end{array}
$$

and

$$
\begin{aligned}
& \left|\phi(t)-E_{0, N}(t)\right| \\
& \leq \frac{4 L R^{2}}{a W} e^{-a t}+\frac{4 L R R^{*}(L+M)}{a a^{*} d_{N} W} . \\
& \left|\phi(t)-E_{0, N}(t)\right| \\
& \leq \frac{4 L R^{* 2}}{a^{*} W^{*}} e^{-a^{*} t}+\frac{4 L R R^{*}(L+M)}{a a^{*} d_{N} W} .
\end{aligned}
$$

Proof. The existence of the 1-periodic solution $\pi(t)$ of the forward Kolmogorov system follows from the exponential

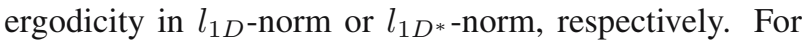
obtaining the first summands in all inequalities, we consider the respective expression on the right-hand side of (9). Namely, we have, instead of $\left\|\mathbf{p}^{*}(s)-\mathbf{p}^{* *}(s)\right\|_{1 D}$, the following quantity:

$$
\begin{aligned}
\left\|\pi(0)-\mathbf{e}_{0}\right\|_{1 D} & =\|\pi(0)\|_{1 D} \\
& =\|\mathbf{q}(0)\|_{1 D} \\
& \leq \limsup _{t \rightarrow \infty}\|\mathbf{q}(t)\|_{1 D},
\end{aligned}
$$

where $\pi(t)$ and $\mathbf{q}(t)$ are the correspondent solutions of (4) and (11). Now we have, instead of (46), the following bound:

$$
\begin{aligned}
& \|\mathbf{q}(t)\|_{1 D} \\
& \leq\|V(t)\|_{1 D}\|\mathbf{q}(0)\|_{1 D} \\
& \quad+\int_{0}^{t}\|V(t, \tau)\|_{1 D}\|\mathbf{f}(\tau)\|_{1 D} \mathrm{~d} \tau \\
& \leq R e^{-a t}\|\mathbf{q}(0)\|_{1 D}+\frac{L R}{a},
\end{aligned}
$$

and

$$
\left\|\pi(0)-\mathbf{e}_{0}\right\|_{1 D} \leq \limsup _{t \rightarrow \infty}\|\mathbf{q}(t)\|_{1 D} \leq \frac{L R}{a} .
$$

Similarly, (46) implies the bound

$$
\left\|\pi(0)-\mathbf{e}_{0}\right\|_{1 D^{*}} \leq \limsup _{t \rightarrow \infty}\|\mathbf{q}(t)\|_{1 D^{*}} \leq \frac{L R^{*}}{a^{*}} .
$$

Finally, the claim follows from the inequalities (10), (19), (34), 35), and 62), 63). 
Remark 1. The best previous bounds contain an additional factor of $t$ on the right-hand sides of (34) and (35), (see Zeifman et al., 2006).

\section{4. $M_{t} / M_{t} / S$ queue}

In this section we deal with a classic queueing model. An $M_{t} / M_{t} / S$ is a stochastic process whose values correspond to the numbers of customers in the system, including any currently in service. Arrivals occur at the rate $\lambda(t)$ according to an inhomogeneous Poisson process and move the process from state $i$ to $i+1$. Service times have a nonstationary exponential distribution with the parameter $\mu(t)$ in the $M_{t} / M_{t} / S$ queue. There are $S$ servers which serve from the front of the queue. If there are less than $S$ jobs, some of the servers will be idle. If there are more than $S$ jobs, the jobs queue in a buffer. The buffer is of infinite size, so there is no limit on the number of customers it can contain. Let $X=$ $X(t), t \geq 0$ be a queue-length process for the $M_{t} / M_{t} / S$ queue. This is a BDP with the birth and death rates $\lambda_{n}(t)=\lambda(t)$ and $\mu_{n}(t)=\min (n, S) \mu(t)$, respectively. There are a number of investigations of this model in a general situation, and, especially, in the case of periodic intensities and for the simplest $M_{t} / M_{t} / 1$ model (see Di Crescenzo and Nobile, 1995; Knessl, 2000; Knessl and Yang, 2002; Mandelbaum and Massey, 1995; Margolius, 2007a; 2007b; Massey and Whitt, 1994; Massey, 2002; Zhang and Coyle, 1991).

Bounds on the rate of convergence, truncations and stability for this process were obtained by Granovsky and Zeifman (2004), Zeifman (1995b; 1995a), Zeifman et al. (2006) as well as Zeifman and Korotysheva (2012). Here we improve estimates of the truncation error.

Assume that there exist $\delta>1$, a function $\theta^{*}(t)$ and positive numbers $R^{*}$ and $a^{*}$ such that

$$
S \mu(t)-\delta^{2} \lambda(t) \geq \theta^{*}(t)
$$

and

$$
e^{-\int_{s}^{t}\left(1-\delta^{-2}\right) \theta^{*}(u) \mathrm{d} u} \leq R^{*} e^{-a^{*}(t-s)},
$$

for any $s$ and $t, 0 \leq s \leq t$.

One can see that for some function $\theta(t)$ and positive numbers $R$ and $a$ the corresponding bounds

$$
S \mu(t)-\delta \lambda(t) \geq \theta(t)
$$

and

$$
e^{-\int_{s}^{t}\left(1-\delta^{-1}\right) \theta(u) \mathrm{d} u} \leq R e^{-a(t-s)},
$$

for any $s$ and $t, 0 \leq s \leq t$, hold, too.

For $S=1$ we can choose $\delta>1$ arbitrarily, and if $S>1$ we suppose $\delta^{2} \leq S /(S-1)$. Note that this assumption is unnecessary. It is formulated here only for ease of computation. If we choose $\delta^{2}>S /(S-1)$, then we obtain another formula for the bounding of $\alpha(t)$ instead of (71).

Set $d_{k}=\delta^{k-1}$. Then

$$
g_{k}=\sum_{n=1}^{k} d_{n}=\frac{\delta^{k}-1}{\delta-1} .
$$

Consider

$$
\begin{aligned}
\alpha_{k}(t)= & \lambda(t)+\min (k+1, S) \mu(t) \\
& -\delta \lambda(t)-\frac{1}{\delta} \min (k, S) \mu(t), \quad k \geq 0 .
\end{aligned}
$$

Then

$$
\alpha_{k}(t) \geq \alpha_{0}(t) \geq \lambda(t)+\mu(t)-\delta \lambda(t)
$$

for $k<S$, and

$$
\alpha_{k}(t) \geq(S \mu(t)-\delta \lambda(t))\left(1-\delta^{-1}\right)
$$

for $k \geq S$.

Hence the inequality $\delta \leq \delta^{2} \leq S /(S-1)$ implies

$$
\begin{aligned}
\alpha(t) & \geq \alpha_{S}(t) \\
& \geq(S \mu(t)-\delta \lambda(t))\left(1-\delta^{-1}\right) \\
& \geq\left(1-\delta^{-1}\right) \theta(t),
\end{aligned}
$$

and we obtain the following theorem.

Theorem 6. The following bounds of truncation for the queue-length process of the $M_{t} / M_{t} / S$ queue hold:

$$
\left\|\mathbf{p}(t)-\mathbf{p}_{N}(t)\right\| \leq \frac{8 L R R^{*}(L+M)}{a a^{*} d_{N}},
$$

for $\mathbf{p}(0)=\mathbf{p}_{N}(0)=\mathbf{e}_{\mathbf{0}}$

$$
\left|E_{0}(t)-E_{0, N}(t)\right| \leq \frac{4 L R R^{*}(L+M)}{a a^{*} d_{N} W},
$$

where $g_{N}=\left(\delta^{N}-1\right) /(\delta-1)$.

Moreover, Theorem 4 is valid for both sequences $\left\{d_{i}\right\}$ and $\left\{d_{i}^{*}\right\}$. Hence we obtain the following theorem.

Theorem 7. The following bounds of truncation for the queue-length process of the $M_{t} / M_{t} / S$ queue hold:

$$
\begin{aligned}
& \left\|\mathbf{p}(t, k)-\mathbf{p}_{N}(t, 0)\right\| \\
& \leq 8 R g_{k} e^{-a t}+\frac{8 L R R^{*}(L+M)}{a a^{*} d_{N}}, \\
& \left\|\mathbf{p}(t, k)-\mathbf{p}_{N}(t, 0)\right\| \\
& \leq 8 R^{*} g_{k}^{*} e^{-a^{*} t}+\frac{8 L R R^{*}(L+M)}{a a^{*} d_{N}},
\end{aligned}
$$


where $\mathbf{p}(0, k)=\mathbf{e}_{\mathbf{k}}, \mathbf{p}_{N}(0,0)=\mathbf{e}_{\mathbf{0}}$,

$$
\begin{array}{r}
\left|E_{k}(t)-E_{0, N}(t)\right| \\
\leq \frac{4 R g_{k}}{W} e^{-a t}+\frac{4 L R R^{*}(L+M)}{a a^{*} d_{N} W}, \\
\left|E_{k}(t)-E_{0, N}(t)\right| \\
\leq \frac{4 R^{*} g_{k}^{*}}{W^{*}} e^{-a^{*} t}+\frac{4 L R R^{*}(L+M)}{a a^{*} d_{N} W}, \\
g_{N}=\frac{\delta^{N}-1}{\delta-1}, \quad g_{N}^{*}=\frac{\delta^{2 N}-1}{\delta^{2}-1} .
\end{array}
$$

Consider the case of 1-periodic arrival and service rates in greater detail. In this case, the conditions 64)-67) appear substantially simpler. Namely, let the following assumption hold:

$$
\int_{0}^{1}(S \mu(t)-\lambda(t)) \mathrm{d} t>0 .
$$

Then

$$
\int_{0}^{1} S \mu(t) \mathrm{d} t>\int_{0}^{1} \lambda(t) \mathrm{d} t .
$$

Hence, for a sufficiently small $\eta>0$,

$$
\int_{0}^{1} S \mu(t) \mathrm{d} t>(1+\eta) \int_{0}^{1} \lambda(t) \mathrm{d} t .
$$

Therefore, setting $\delta_{0}^{2}=(1+\eta)>1$, we have

$$
\int_{0}^{1}\left(S \mu(t)-\delta_{0}^{2} \lambda(t)\right) \mathrm{d} t>0 .
$$

Then

$$
\int_{0}^{1}\left(S \mu(t)-\delta_{0} \lambda(t)\right) \mathrm{d} t>0,
$$

and we can choose

$$
\theta_{0}^{*}(t)=S \mu(t)-\delta_{0}^{2} \lambda(t)
$$

and

$$
\theta_{0}(t)=S \mu(t)-\delta_{0} \lambda(t)
$$

Therefore, we obtain

$$
\begin{array}{r}
\alpha_{0}(t)=\left(1-\delta_{0}^{-1}\right) \theta_{0}(t), \quad a_{0}=\int_{0}^{1} \alpha_{0}(t) \mathrm{d} t, \\
R_{0}=e^{K_{0}}, \quad K_{0}=\sup _{|t-s| \leq 1} \int_{s}^{t} \alpha_{0}(u) \mathrm{d} u .
\end{array}
$$

Similarly, we have

$$
\begin{array}{r}
\alpha_{0}^{*}(t)=\left(1-\delta_{0}^{-2}\right) \theta_{0}^{*}(t), \quad a_{0}^{*}=\int_{0}^{1} \alpha_{0}^{*}(t) \mathrm{d} t, \\
R_{0}^{*}=e^{K_{0}^{*}}, \quad K_{0}^{*}=\sup _{|t-s| \leq 1} \int_{s}^{t} \alpha_{0}^{*}(u) \mathrm{d} u .
\end{array}
$$

Theorem 8. Let the arrival and service rates in the $M_{t} / M_{t} / S$ queue be 1-periodic, and let (78) hold. Then there exist a limiting 1-periodic probability distribution $\pi(t)$, say, and the respective limiting 1-periodic mean $\phi(t)$ for the queue-length process $X(t)$, and the following bounds hold:

$$
\begin{array}{r}
\left\|\pi(t)-\mathbf{p}_{N}(t, 0)\right\| \\
\leq \frac{4 L R_{0}^{2}}{a_{0}} e^{-a_{0} t}+\frac{8 L R_{0} R_{0}^{*}(L+M)}{a_{0} a_{0}^{*} d_{N}}, \\
\left\|\pi(t)-\mathbf{p}_{N}(t, 0)\right\| \\
\leq \frac{4 L R_{0}^{* 2}}{a_{0}^{*}} e^{-a_{0}^{*} t}+\frac{8 L R_{0} R_{0}^{*}(L+M)}{a_{0} a_{0}^{*} d_{N}},
\end{array}
$$

and

$$
\begin{array}{r}
\left|\phi(t)-E_{0, N}(t)\right| \\
\leq \frac{4 L R_{0}^{2}}{a_{0} W} e^{-a_{0} t}+\frac{4 L R_{0} R_{0}^{*}(L+M)}{a_{0} a_{0}^{*} d_{N} W}, \\
\left|\phi(t)-E_{0, N}(t)\right| \\
\leq \frac{4 L R_{0}^{* 2}}{a_{0}^{*} W^{*}} e^{-a_{0}^{*} t}+\frac{4 L R_{0} R_{0}^{*}(L+M)}{a_{0} a_{0}^{*} d_{N} W} .
\end{array}
$$

\section{Examples}

5.1. Example 1. Let $X=X(t), t \geq 0$ be a queue-length process for a concrete $M_{t} / M_{t} / S$ with $S=$ $2, \lambda(t)=1+\sin 0.02 \pi t, \mu(t)=4+2 \cos 0.02 \pi t$. This example of a queueing system with periodical intensity functions with a sufficiently large period $T=100$ was considered by Zeifman et al. (2006). Now we can obtain essentially more accurate bounds.

Remark 2. This example deals with the situation that can happen if the intensities of arrivals and services have different periods. As this is so if these periods are commensurable, the limit characteristics will also be periodic, although with a rather large period. Here $L=2$, $M=6$.

Set $\delta_{0}=\sqrt{2}$. Then $d_{0, k}=2^{\frac{k-1}{2}}$ and $d_{0, k}^{*}=2^{k-1}$. We have

$$
\begin{gathered}
W=\inf _{i \geq 1} \frac{d_{i}}{i}=\frac{2}{3}, \\
W^{*}=\inf _{i \geq 1} \frac{d_{i}^{*}}{i}=1, \\
g_{N}=\frac{2^{N / 2}-1}{\sqrt{2}-1}, \quad g_{N}^{*}=2^{N}-1 .
\end{gathered}
$$

Further,

$$
\alpha_{0}(t)=(1-1 / \sqrt{2})(2 \mu(t)-\sqrt{2} \lambda(t)),
$$




$$
\begin{aligned}
& a_{0}=9-5 \sqrt{2}>1.5, \\
\alpha_{0}^{*}(t)= & 0.5(2 \mu(t)-2 \lambda(t)) \\
= & 3+2 \cos 0.02 \pi t-\sin 0.02 \pi t, \\
a^{*}= & 3 .
\end{aligned}
$$

Hence we obtain

$$
K_{0}=\sup _{|t-s| \leq 100} \int_{s}^{t} \alpha_{0}(u) \mathrm{d} u=\frac{100(3-\sqrt{2})}{\pi},
$$

and

$$
R_{0}=\exp \frac{100(3-\sqrt{2})}{\pi}<e^{60} .
$$

Similarly,

$$
K_{0}^{*}=\sup _{|t-s| \leq 100} \int_{s}^{t} \alpha_{0}^{*}(u) \mathrm{d} u=\frac{300}{\pi},
$$

and

$$
R_{0}^{*} \leq e^{\frac{300}{\pi}}<e^{100} .
$$

Therefore the inequalities (74) and (76) of Theorem 7 imply the following statement.

Proposition 1. The following bounds hold:

$$
\begin{aligned}
& \left\|\mathbf{p}(k, t)-\mathbf{p}_{N}(0, t)\right\| \\
& \quad \leq 2^{4+k / 2} \cdot e^{60-1.5 t}+\frac{e^{160}}{10 \cdot 2^{\frac{N-17}{2}}}
\end{aligned}
$$

and

$$
\begin{aligned}
\mid E_{k}(t) & -E_{0, N}(t) \mid \\
& \leq \frac{1+\sqrt{2}}{2}\left\|\mathbf{p}(k, t)-\mathbf{p}_{N}(0, t)\right\| \\
& <2^{\frac{k+9}{2}} \cdot e^{60-1.5 t}+\frac{e^{160}}{10 \cdot 2^{\frac{N-18}{2}}},
\end{aligned}
$$

for any $k, N$ and any $t \geq 0$.

Further, we can apply Theorem 8

Proposition 2. The queue-length process for the model has a limiting 1-periodic probability distribution $\pi(t)$, the respective limiting 1-periodic mean $\phi(t)$, and the following bounds hold:

$$
\begin{aligned}
\left\|\pi(t)-\mathbf{p}_{N}(0, t)\right\| & \\
& \leq 16 \cdot e^{119-1.5 t}+\frac{e^{160}}{10 \cdot 2^{\frac{N-17}{2}}},
\end{aligned}
$$

and

$$
\left|\phi(t)-E_{0, N}(t)\right| \leq 8 \cdot e^{120-1.5 t}+\frac{e^{160}}{10 \cdot 2^{\frac{N-18}{2}}},
$$

for any $N$ and any $t \geq 0$.
These bounds can be used for the study and construction of limiting characteristics of the queue, as was firstly shown by Zeifman et al. (2006).

Particularly, for $N \geq 550, t \geq 150$ and the initial conditions $k \leq 400$, we obtain error bounds for all characteristics less than $10^{-10}$.

Note that the best of previously known estimates (see Zeifman et al., 2006, Example 2(iii)) give us (even for $k=$ 0 ) significantly poorer estimates $N=945$ and interval $[418,518]$, and the error bound $10^{-8}$.

The behaviour of probabilistic characteristics of the queue-length process is shown in the figures below.

Remark 3. All the characteristics mentioned above (state probabilities, mathematical expectations, etc.) are found in one and the same way, namely, by solving the Cauchy problem with an appropriate initial condition for the truncated Kolmogorov system by the Runge-Kutta method of the fourth order of accuracy. Moreover, as was shown, the dimensionality of the corresponding system appears to be rather moderate. Further, if the figure depicts a characteristic on the interval $[a, a+T]$, then the Cauchy problem is solved on $[0, a+T]$. Furthermore, the figures show that the rate of convergence to the corresponding limit characteristics is sufficiently large so that the parameter $a$ can be chosen not so large.

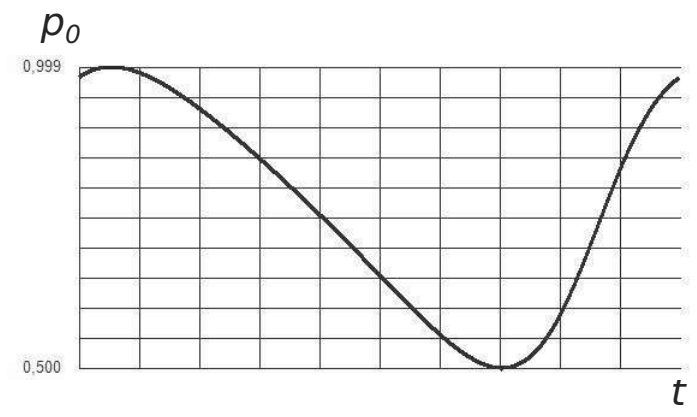

Fig. 1. Probability $\operatorname{Pr}(X(t)=0 \mid X(0)=k)$ of an empty queue for $k=0$ and $t \in[70,170]$.

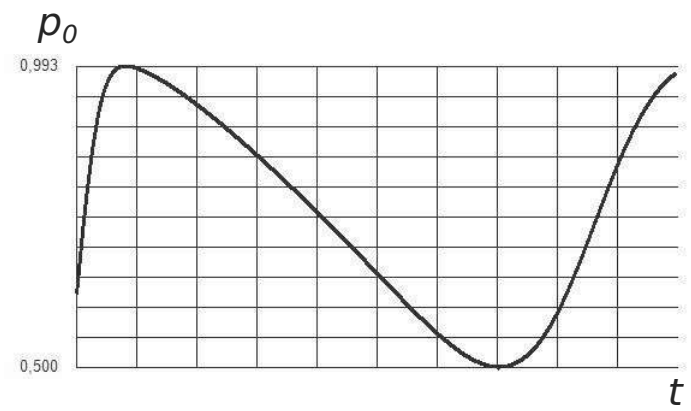

Fig. 2. Probability $\operatorname{Pr}(X(t)=0 \mid X(0)=k)$ of an empty queue for $k=400$ and $t \in[70,170]$. 


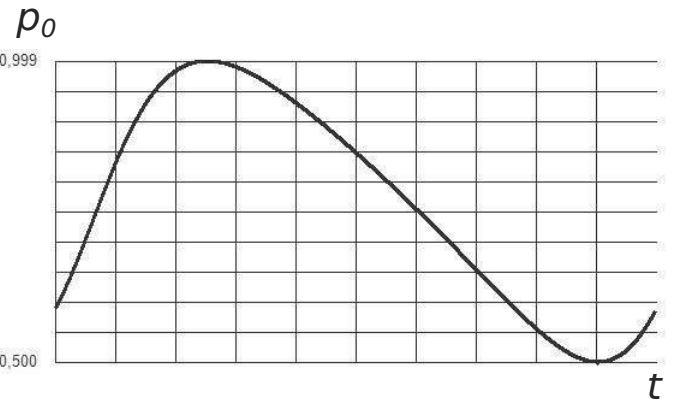

Fig. 3. Probability $\operatorname{Pr}(X(t)=0 \mid X(0)=k)$ of an empty queue for $k=0$ and $t \in[150,250]$.

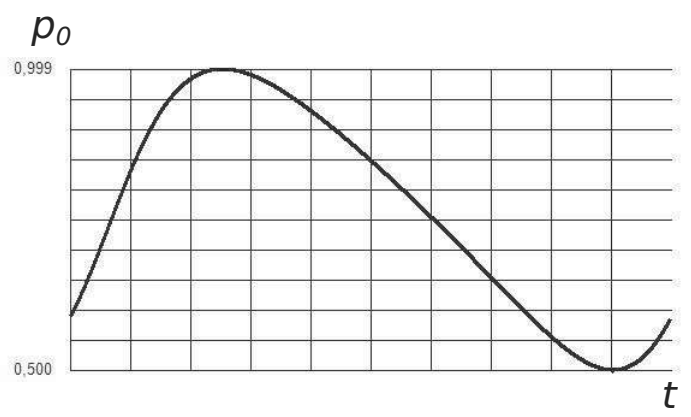

Fig. 4. Probability $\operatorname{Pr}(X(t)=0 \mid X(0)=k)$ of an empty queue for $k=400$ and $t \in[150,250]$.

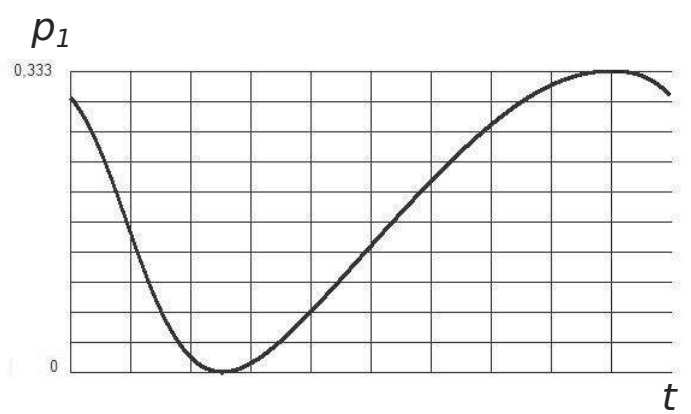

Fig. 5. Probability $\operatorname{Pr}(X(t)=1 \mid X(0)=k)$ for $k=0$ and $t \in$ $[150,250]$.

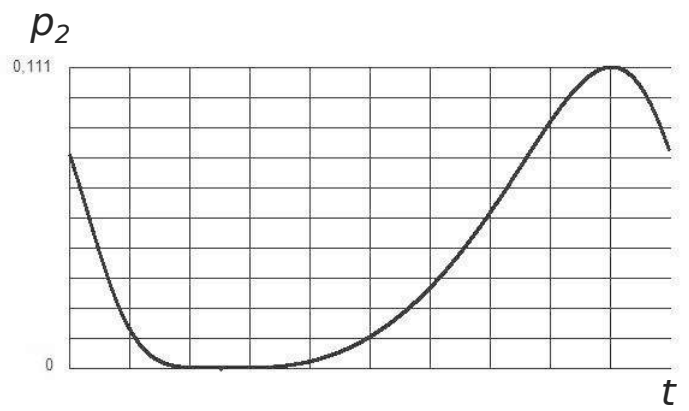

Fig. 6. Probability $\operatorname{Pr}(X(t)=2 \mid X(0)=k)$ for $k=0$ and $t \in$ $[150,250]$.

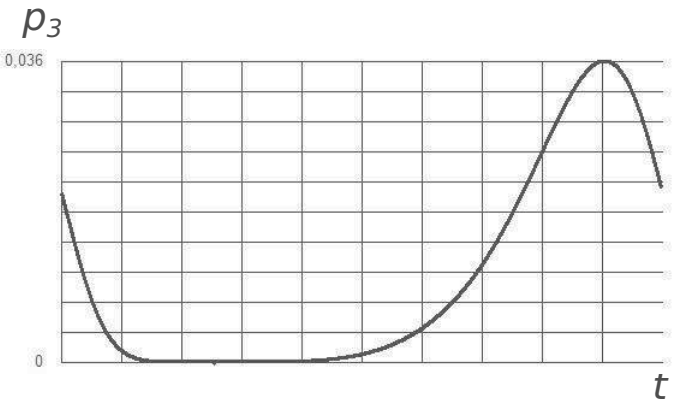

Fig. 7. Probability $\operatorname{Pr}(X(t)=3 \mid X(0)=k)$ for $k=0$ and $t \in$ [150, 250].

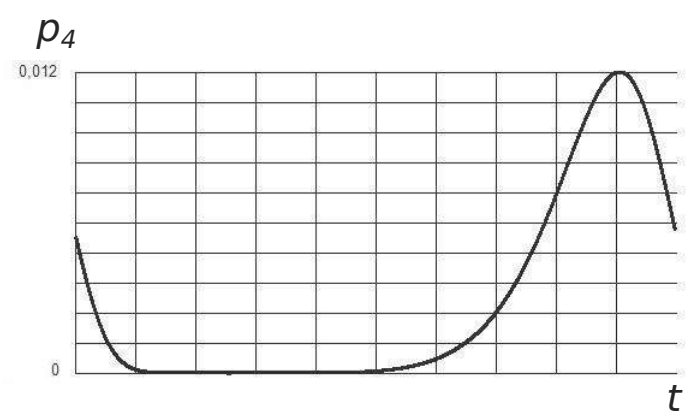

Fig. 8. Probability $\operatorname{Pr}(X(t)=4 \mid X(0)=k)$ for $k=0$ and $t \in$ $[150,250]$.

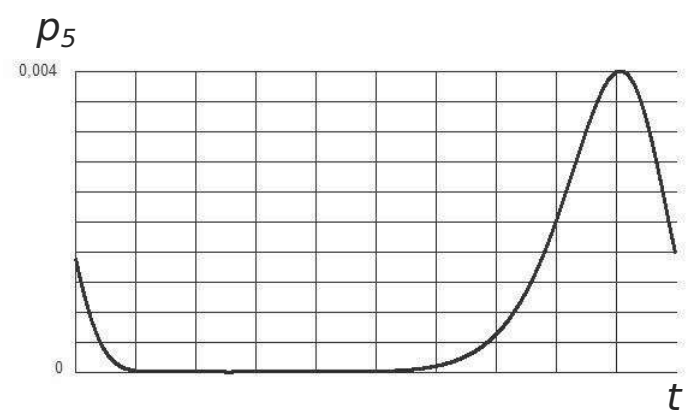

Fig. 9. Probability $\operatorname{Pr}(X(t)=5 \mid X(0)=k)$ for $k=0$ and $t \in$ $[150,250]$.

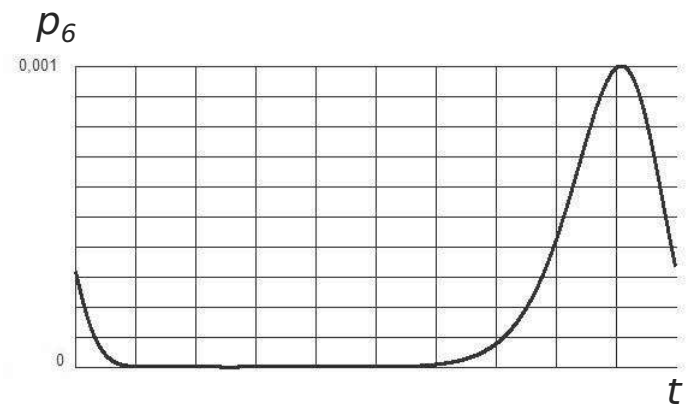

Fig. 10. Probability $\operatorname{Pr}(X(t)=6 \mid X(0)=k)$ for $k=0$ and $t \in[150,250]$. 
Remark 4. One can see in Figs. 3 and 4 that they give us very good approximations of the real limiting probability of an empty queue.

Remark 5. In Figs. 5-10 one can see the limiting probability of the corresponding length of the queue.

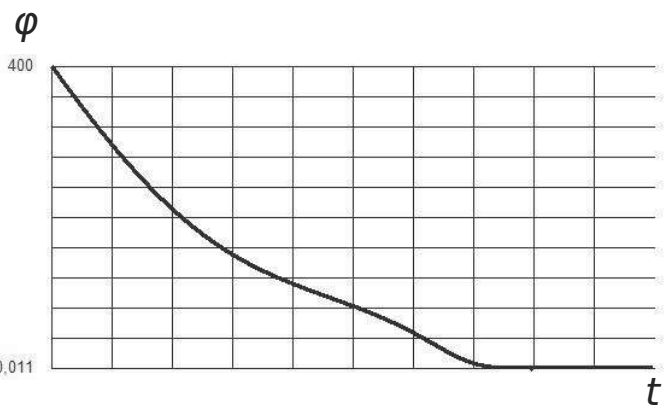

Fig. 11. Mathematical expectation of the length of the queue $E_{400}(t), k=400$ and $t \in[0,100]$.

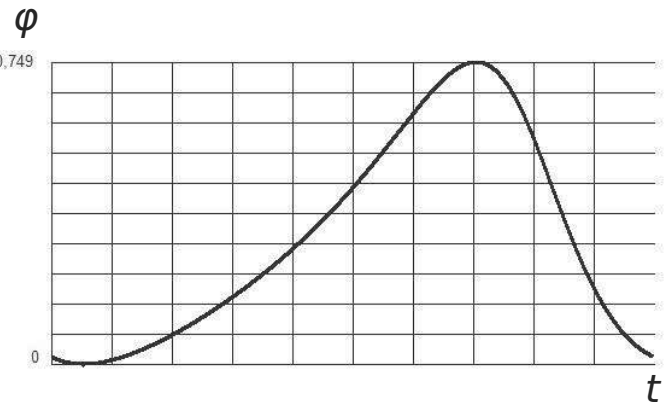

Fig. 12. Mathematical expectation of the length of the queue $E_{0}(t), k=0$ and $t \in[70,170]$

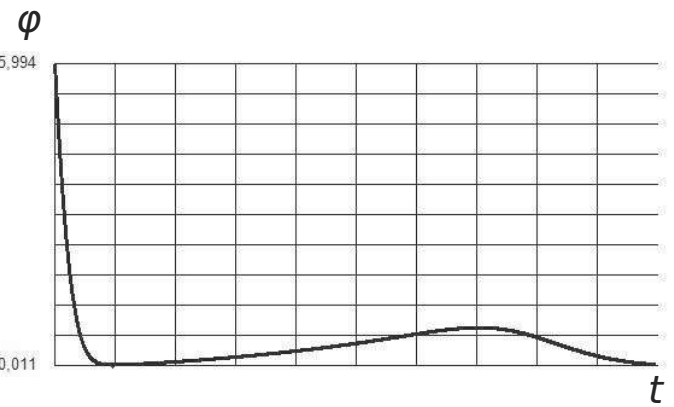

Fig. 13. Mathematical expectation of the length of the queue $E_{400}(t), k=400$ and $t \in[70,170]$.

Remark 6. In Figs. 14 and 15 one can see that they give us very good approximation of the real limiting mean (mathematical expectation) of the length of a queue.

5.2. Example 2. Let $X=X(t), t \geq 0$ be now a queue-length process for $M_{t} / M_{t} / S$ with $S=10^{12}$,

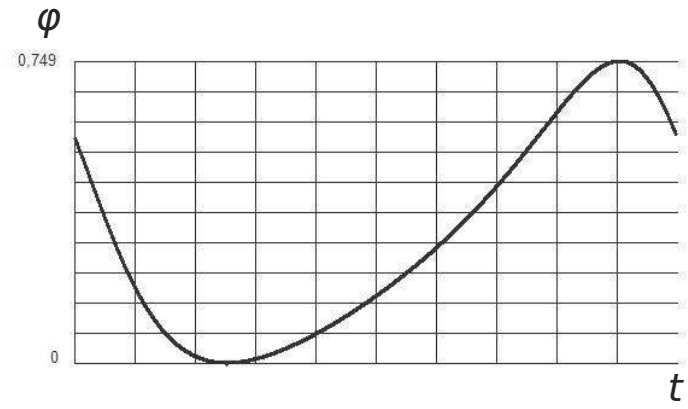

Fig. 14. Mathematical expectation of the length of the queue $E_{0}(t), k=0$ and $t \in[150,250]$.

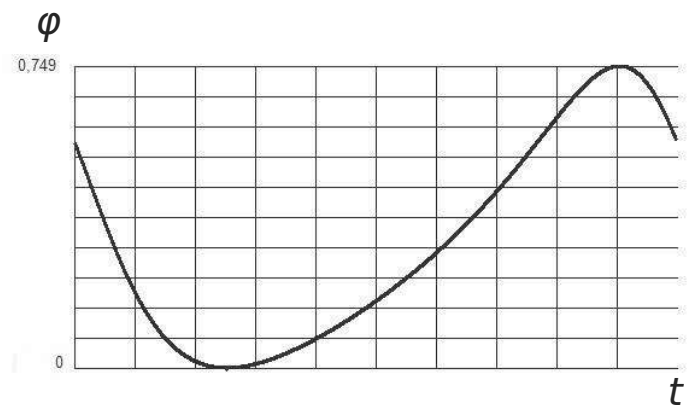

Fig. 15. Mathematical expectation of the length of the queue $E_{400}(t), k=400$ and $t \in[150,250]$.

$\lambda(t)=1+\sin 2 \pi t, \mu(t)=3+2 \cos 2 \pi t . \quad$ This example and its analogue for a queueing system with group services was considered by Zeifman et al. (2013b).

Now we consider only an ordinary "classic" queueing model and obtain its main limiting characteristics. Here $L=2, M=5 \cdot 10^{12}$. Put $\delta_{0}=\sqrt{2}$. Then $d_{0, k}=2^{\frac{k-1}{2}}$ and $d_{0, k}^{*}=2^{k-1}$. We have

$$
\begin{aligned}
W & =\inf _{i \geq 1} \frac{d_{i}}{i}=\frac{2}{3}, \\
W^{*} & =\inf _{i \geq 1} \frac{d_{i}^{*}}{i}=1, \\
g_{N} & =\frac{2^{N / 2}-1}{\sqrt{2}-1}, \\
g_{N}^{*} & =2^{N}-1 .
\end{aligned}
$$

Further,

$$
\begin{gathered}
\alpha_{0}(t)=\mu(t)-(\sqrt{2}-1) \lambda(t), \\
a_{0}=4-\sqrt{2}>2, \\
\alpha_{0}^{*}(t)=\mu(t)-\lambda(t), \quad a^{*}=2 .
\end{gathered}
$$

Hence we obtain

$$
K_{0}=\sup _{|t-s| \leq 100} \int_{s}^{t} \alpha_{0}(u) \mathrm{d} u=\frac{1+\sqrt{2}}{\pi},
$$


and

$$
R_{0}=\exp \left(\frac{1+\sqrt{2}}{\pi}\right)<e
$$

Similarly,

$$
K_{0}^{*}=\sup _{|t-s| \leq 100} \int_{s}^{t} \alpha_{0}^{*}(u) \mathrm{d} u=\frac{3}{\pi},
$$

and $R_{0}^{*} \leq e^{\frac{3}{\pi}}<e$

Therefore, the inequalities (74) and (76) of Theorem 7 imply the following statement.

Proposition 3. The following bounds hold:

$$
\left\|\mathbf{p}(k, t)-\mathbf{p}_{N}(0, t)\right\| \leq 2^{k / 2} \cdot e^{4-2 t}+\frac{e^{2} \cdot 10^{13}}{2^{\frac{N-3}{2}}}
$$

and

$$
\left|E_{k}(t)-E_{0, N}(t)\right| \leq 2^{(k+2) / 2} \cdot e^{4-2 t}+\frac{3 e^{2} \cdot 10^{13}}{2^{\frac{N-1}{2}}}
$$

for any $k, N$ and any $t \geq 0$.

Further, Theorem 8 implies the following statement.

Proposition 4. A queue-length process for the model has a limiting 1-periodic probability distribution $\pi(t)$, the respective limiting 1-periodic mean $\phi(t)$, and the following bounds hold:

$$
\left\|\pi(t)-\mathbf{p}_{N}(0, t)\right\| \leq 4 \cdot e^{2-2 t}+\frac{e^{2} \cdot 10^{13}}{2^{\frac{N-3}{2}}}
$$

and

$$
\left|\phi(t)-E_{0, N}(t)\right| \leq 6 \cdot e^{2-2 t}+\frac{3 e^{2} \cdot 10^{13}}{2^{\frac{N-1}{2}}}
$$

for any $N$ and any $t \geq 0$.

Here for $N \geq 170, t \geq 85$, and the initial conditions $k \leq N=170$ we obtain error bounds for all characteristics less than $10^{-10}$.

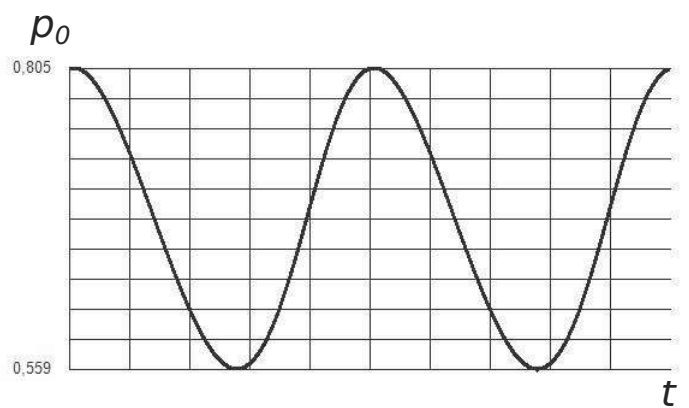

Fig. 16. Probability $\operatorname{Pr}(X(t)=0 \mid X(0)=k)$ of an empty queue for $k=0$ and $t \in[3,5]$.

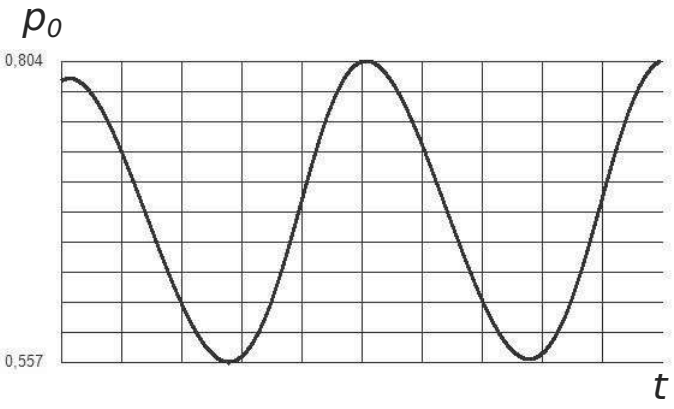

Fig. 17. Probability $\operatorname{Pr}(X(t)=0 \mid X(0)=k)$ of an empty queue for $k=170$ and $t \in[3,5]$

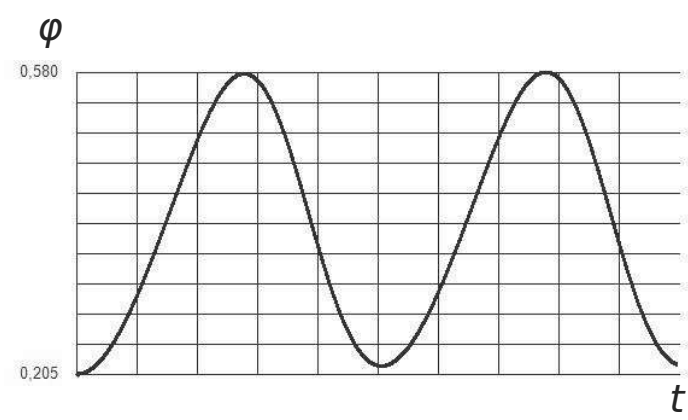

Fig. 18. Mathematical expectation of the length of the queue $E_{k}(t), k=0$ and $t \in[1,3]$.

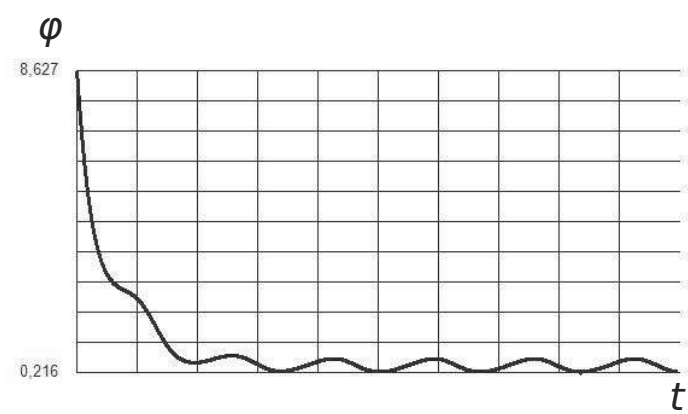

Fig. 19. Mathematical expectation of the length of the queue $E_{k}(t), k=170$ and $t \in[1,7]$.

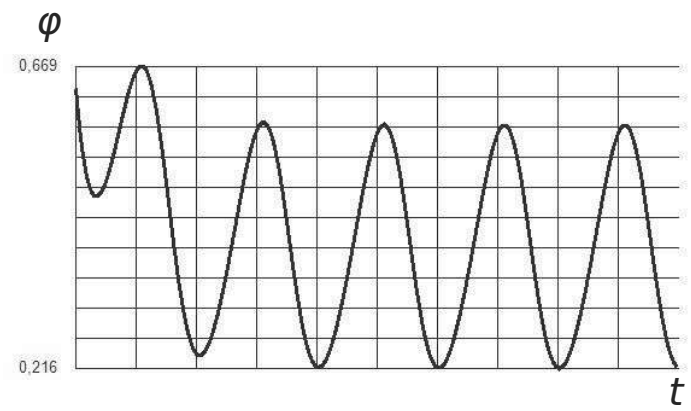

Fig. 20. Mathematical expectation of the length of the queue $E_{k}(t), k=170$ and $t \in[2,7]$. 
ames

516

A. Zeifman et al.

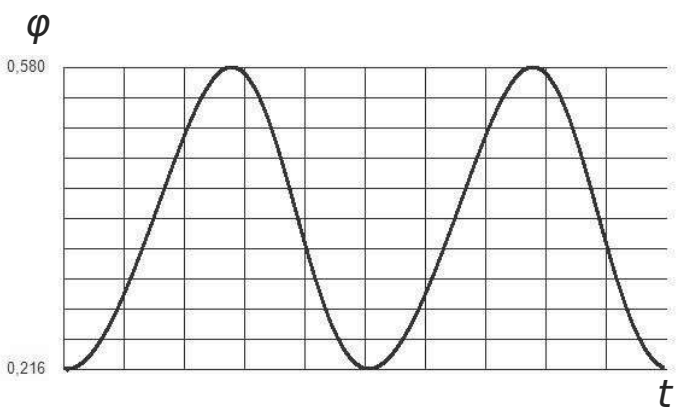

Fig. 21. Mathematical expectation of the length of the queue $E_{k}(t), k=0$ and $t \in[3,5]$.

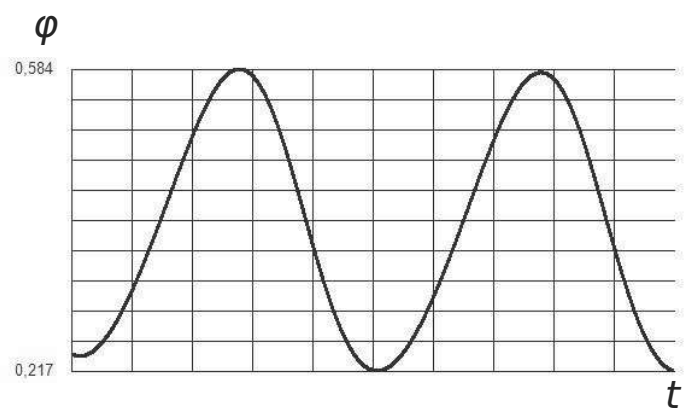

Fig. 22. Mathematical expectation of the length of the queue $E_{k}(t), k=170$ and $t \in[3,5]$.

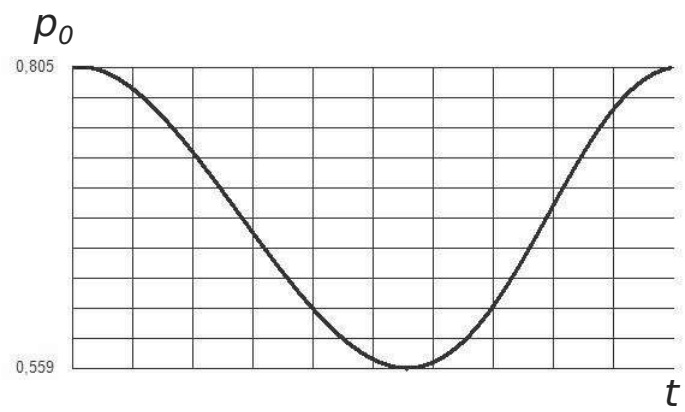

Fig. 23. Approximation of the limiting probability of an empty queue $\operatorname{Pr}(X(t)=0 \mid X(0)=k)$ for $k=0$ and $t \in$ $[85,86]$.

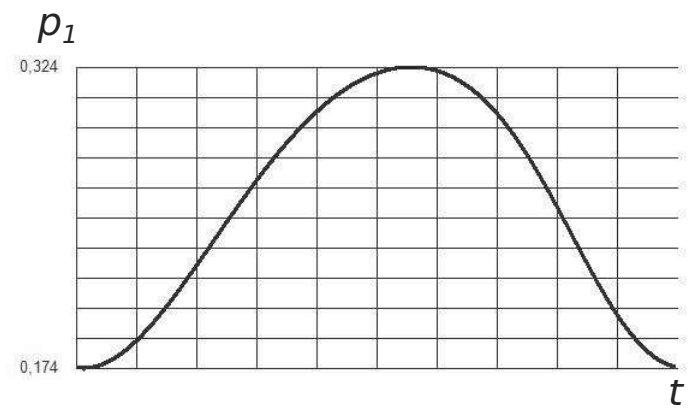

Fig. 24. Approximation of the limiting probability $\operatorname{Pr}(X(t)=1 \mid X(0)=k)$ for $k=0$ and $t \in[85,86]$.

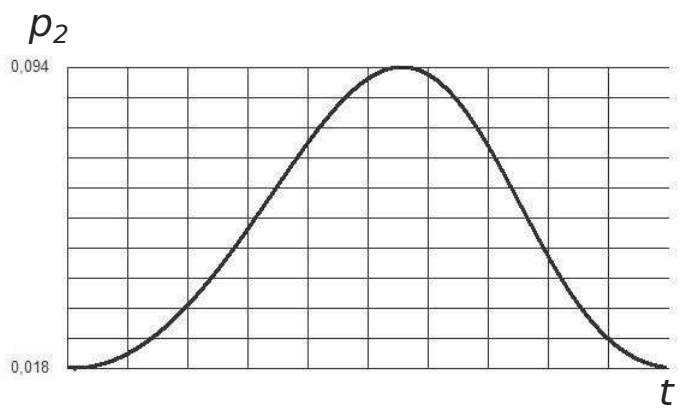

Fig. 25. Approximation of the limiting probability $\operatorname{Pr}(X(t)=2 \mid X(0)=k)$ for $k=0$ and $t \in[85,86]$.

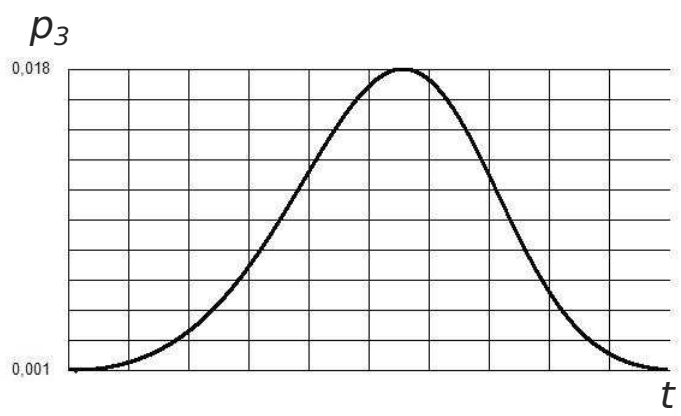

Fig. 26. Approximation of the limiting probability $\operatorname{Pr}(X(t)=3 \mid X(0)=k)$ for $k=0$ and $t \in[85,86]$.

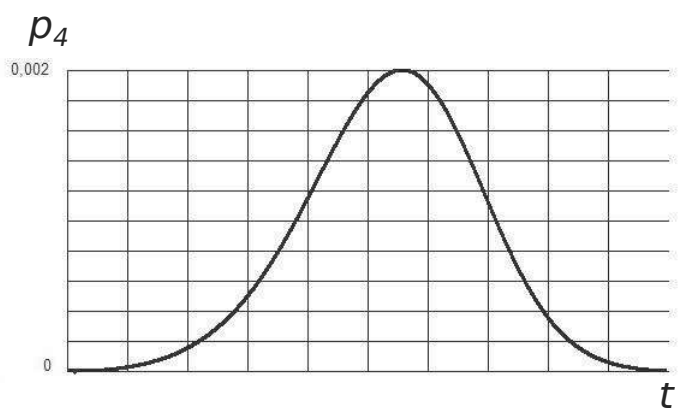

Fig. 27. Approximation of the limiting probability $\operatorname{Pr}(X(t)=4 \mid X(0)=k)$ for $k=0$ and $t \in[85,86]$.

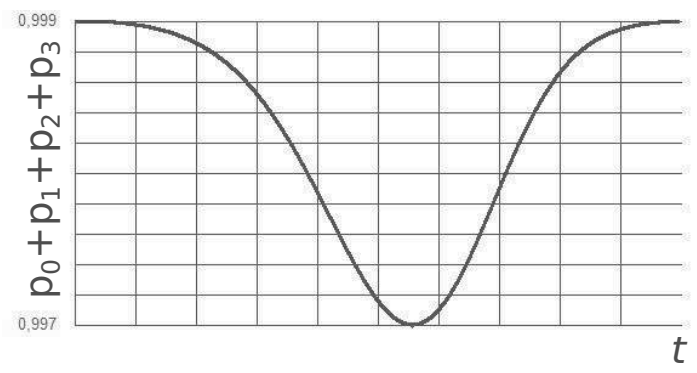

Fig. 28. Approximation of the limiting probability $\operatorname{Pr}(X(t) \leq 3 \mid X(0)=k)$ for $k=0$ and $t \in[85,86]$. 


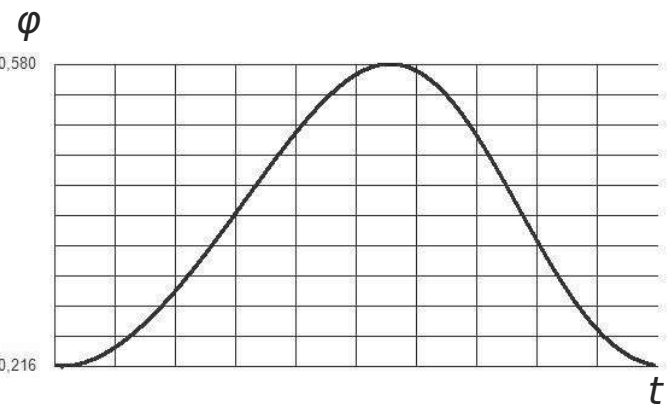

Fig. 29. Approximation of the limiting mathematical expectation of the length of the queue $\phi(t)$ by $E_{k}(t), k=0$ and $t \in[85,86]$.

\section{Conclusion}

In this paper, we investigated a class of weakly ergodic inhomogeneous birth and death processes and obtained uniform (in time) error bounds of truncation. Our approach also guarantees that we can find limiting characteristics approximately with an arbitrary fixed error, see the detailed discussion by Zeifman et al. (2006). Moreover, we can find the limiting characteristics for any number of servers $S$; see the respective example for $S=10^{12}$ by Zeifman et al. (2013b). Arbitrary intensity functions instead of periodic ones can be considered in the same manner. Finally, we would like to remark that all of our results can be applied to birth and death process with catastrophes; see perturbation bounds in the work of Zeifman and Korotysheva (2012) and the bounds on the rate of convergence given by Zeifman et al. (2013a).

\section{Acknowledgment}

The authors are grateful to the referees for their helpful comments and suggestions. This research was supported by the Russian Foundation for Basic Research (projects 12-07-00109, 12-07-00115, 13-07-00223, 14-07-00041).

\section{References}

Daleckij, J.L. and Krein, M.G. (1974). Stability of Solutions of Differential Equations in Banach Space, American Mathematical Society, Providence, RI.

Di Crescenzo, A., Giorno, V., Kumar, B.K. and Nobile, A.G. (2012). A double-ended queue with catastrophes and repairs, and a jump-diffusion approximation, Methodology and Computing in Applied Probability 14(4): 937-954, DOI:10.1007/s11009-011-9214-2.

Di Crescenzo, A., Giorno, V., Nobile, A.G. and Ricciardi, L.M. (2003). On the $M / M / 1$ queue with catastrophes and its continuous approximation, Queueing Systems 43(4): 329-347, DOI:10.1023/A:1023261830362.

Di Crescenzo, A. and Nobile, A.G. (1995). Diffusion approximation to a queueing system with time dependent arrival and service rates, Queueing systems 19(1): 41-62, DOI:10.1007/BF01148939.

Granovsky, B. and Zeifman, A. (2004). Nonstationary queues: Estimation of the rate of convergence, Queueing Systems 46(3): 369-388, DOI:10.1023/B:QUES.0000027991.19758.b4.

Knessl, C. (2000). Exact and asymptotic solutions to a PDE that arises in time-dependent queues, Advances in Applied Probability 32(1): 256-283.

Knessl, C. and Yang, Y.P. (2002). An exact solution for an $M(t) / M(t) / 1$ queue with time-dependent arrivals and service, Advances in Applied Probability 40(3): 233-248, DOI:10.1023/A:1014786928831.

Mandelbaum, A. and Massey, W. (1995). Strong approximations for time-dependent queues, Mathematics of Operations Research 20(1): 33-64, DOI:10.1287/moor.20.1.33.

Margolius, B. (2007a). Periodic solution to the time-inhomogeneous multi-server Poisson queue, Operations Research Letters 35(1): 125-138, DOI:10.1016/j.orl.2005.12.008.

Margolius, B. (2007b). Transient and periodic solution to the time-inhomogeneous quasi-birth death process, Queueing Systems 56(3): 183-194, DOI:10.1007/s11134-007-9027-8.

Massey, W. (2002). The analysis of queues with time-varying rates for telecommunication models, Telecommunication Systems 21(2): 173-204, DOI:10.1023/A:1020990313587.

Massey, W. and Pender, J. (2013). Gaussian skewness approximation for dynamic rate multi-server queues with abandonment, Queueing Systems 75(2): 243-277.

Massey, W. and Whitt, W. (1994). On analysis of the modified offered-load approximation for the nonstationary Erlang loss model, Annals of Applied Probability 4(4): 1145-1160, DOI:10.1214/aoap/1177004908.

Olwal, T.O., Djouani, K., Kogeda, O.P. and van Wyk, B.J.V (2012). Joint queue-perturbed and weakly coupled power control for wireless backbone networks, International Journal of Applied Mathematics and Computer Science 22(3): 749-764, DOI: 10.2478/v10006-012-0056-z.

Tan, X., Knessl, C. and Yang, Y.P. (2013). On finite capacity queues with time dependent arrival rates, Stochastic Processes and their Applications 123(6): 2175-2227, DOI:10.1016/j.spa.2013.02.002.

Van Doorn, E.A., Zeifman, A.I. and Panfilova, T.L. (2010). Bounds and asymptotics for the rate of convergence of birth-death processes, Theory of Probability and Its Applications 54(1): 97-113, DOI:10.1137/S0040585X97984097.

Zeifman, A. (1995a). Upper and lower bounds on the rate of convergence for nonhomogeneous birth and death processes, Stochastic Processes and Their Applications 59(1): 157-173, DOI:10.1016/0304-4149(95)00028-6.

Zeifman, A.I. (1985). Stability for continuous-time nonhomogeneous Markov chains, in V.V. Kalashnikov and V.M. Zolotarev (Eds.), Stability Problems for 
Stochastic Models, Lecture Notes in Mathematics, Vol. 1155, Springer, Berlin/Heidelberg, pp. 401-414, DOI: $10.1007 / \mathrm{BFb} 0074830$.

Zeifman, A.I. (1988). Truncation error in a birth and death system, USSR Computational Mathematics and Mathematical Physics 28(6): 210-211, DOI:10.1016/0041-5553(88)90068-7.

Zeifman, A.I. (1995b). On the estimation of probabilities for birth and death processes, Journal of Applied Probability 32(3): 623-634.

Zeifman, A., Korolev, V., Satin, Y., Korotysheva, A. and Bening, V. (2014). Perturbation bounds and truncations for a class of Markovian queues, Queueing Systems 76(2): 205-221, DOI:10.1007/s11134-013-9388-0.

Zeifman, A. and Korotysheva, A. (2012). Perturbation bounds for $M_{t} / M_{t} / N$ queue with catastrophes, Stochastic Models 281(1): 49-62, DOI:10.1080/15326349.2011.614900.

Zeifman, A., Leorato, S., Orsingher, E., Satin, Y. and Shilova, G. (2006). Some universal limits for nonhomogeneous birth and death processes, Queueing Systems 52(2): 139-151, DOI:10.1007/s11134-006-4353-9.

Zeifman, A., Satin, Y. and Panfilova, T. (2013a). Limiting characteristics for finite birth-death-catastrophe processes, Mathematical Biosciences 245(1): 96-102, DOI:10.1016/j.mbs.2013.02.009.

Zeifman, A., Satin, Y., Shilova, G., Korolev, V., Bening, V. and Shorgin, S. (2013b). On $M_{t} / M_{t} / S$ type queue with group services, Proceedings of the 27th European Conference on Modeling and Simulation, Aalesund, Norway, pp. 604-609.

Zhang, J. and Coyle, E.J.J. (1991). The transient solution of time-dependent $M / M / L$ queues, IEEE Transactions on Information Theory 37(6): 1690-1696, DOI:10.1109/18.104335.

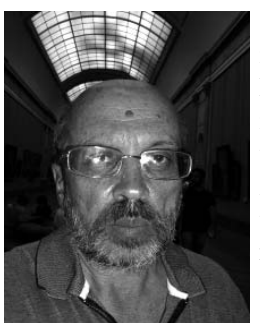

Alexander Zeifman is a Doctor of Science in physics and mathematics, a professor, the head of the Department of Applied Mathematics (Vologda State University), a senior scientist (Institute of Problems of Informatics, Russian Academy of Sciences) a principal scientist (Institute of Socio-Economic Development of Territories, Russian Academy of Sciences).

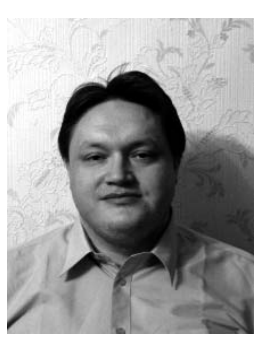

Yacov Satin is a Candidate of Science (Ph.D.) in physics and mathematics, and an associate professor (Vologda State University).

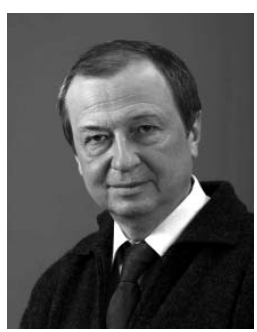

Victor Korolev is a Doctor of Science in physics and mathematics, a professor, the head of the Department of Mathematical Statistics (Faculty of Computational Mathematics and Cybernetics, M.V. Lomonosov Moscow State University), a leading scientist (Institute of Problems of Informatics, Russian Academy of Sciences).

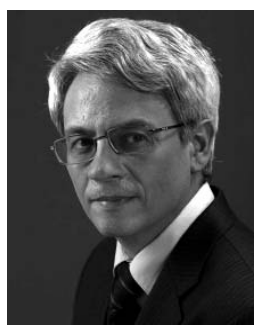

Sergey Shorgin is a Doctor of Science in physics and mathematics, a professor, a deputy director (Institute of Problems of Informatics, Russian Academy of Sciences)

Received: 30 July 2013

Revised: 3 February 2014 